\title{
L'expérience du personnel enseignant formé à l'étranger dans les écoles de Montréal. Apports et enseignements d'une recherche collaborative
}

\section{The experience of internationally educated teachers in} Montreal schools. Contributions and lessons from a collaborative study

\section{La experiencia del personal docente formado en el extranjero en las escuelas de Montreal. Aportes y enseñanzas de una investigación colaborativa}

\author{
Joëlle Morrissette et Didier Demazière
}

\section{Volume 46, numéro 2, automne 2018}

Pluralisme, équité et rapports ethniques dans la formation du personnel des milieux éducatifs

URI : https://id.erudit.org/iderudit/1055568ar DOI : https://doi.org/10.7202/1055568ar

Aller au sommaire du numéro

Éditeur(s)

Association canadienne d'éducation de langue française

ISSN

1916-8659 (numérique)

Découvrir la revue

Citer cet article

Morrissette, J. \& Demazière, D. (2018). L'expérience du personnel enseignant formé à l'étranger dans les écoles de Montréal. Apports et enseignements d'une recherche collaborative. Éducation et francophonie, 46(2), 189-207. https://doi.org/10.7202/1055568ar

\section{Résumé de l'article}

L'intégration professionnelle du personnel enseignant formé à l'étranger travaillant dans les écoles montréalaises est un enjeu d'importance. S’il suit une courte formation, c'est surtout au coeur des situations de travail qu'il réapprend le métier. Jusqu'à présent, la focale a surtout été mise sur les difficultés d'intégration des membres de ce personnel enseignant dans leur nouvel environnement de travail. À rebours, une approche interactionniste de l'intégration d'un groupe minoritaire à un groupe majoritaire conduit plutôt à considérer leurs expériences comme le produit de conventions professionnelles, propres aux contextes de travail, qui s'entrechoquent. La réalisation d'entretiens individuels et collectifs avec des enseignantes et des enseignants formés à l'étranger et avec certains de leurs partenaires a permis une coanalyse des événements marquants ayant jalonné leurs premières expériences dans les écoles montréalaises, et traduisant des tensions avec leurs expériences d'enseignement antérieures à la migration. Dans cette contribution, nous mettons l'accent sur les chocs entre conventions tacites définissant le travail enseignant et en examinons trois dimensions : les relations de travail, l'expertise au fondement du métier et les sources de la reconnaissance professionnelle. Enfin, nous discutons de l'intérêt du dispositif collaboratif pour les participantes et les participants et, au-delà, pour les personnels éducatifs, notamment les directions d'établissement qui accueillent du personnel enseignant de différents horizons dans leur équipe-école.
Tous droits réservés (C) Association canadienne d'éducation de langue française, 2018
Cocument est protégé par la loi sur le droit d'auteur. L'utilisation des services d’Érudit (y compris la reproduction) est assujettie à sa politique d'utilisation que vous pouvez consulter en ligne. 


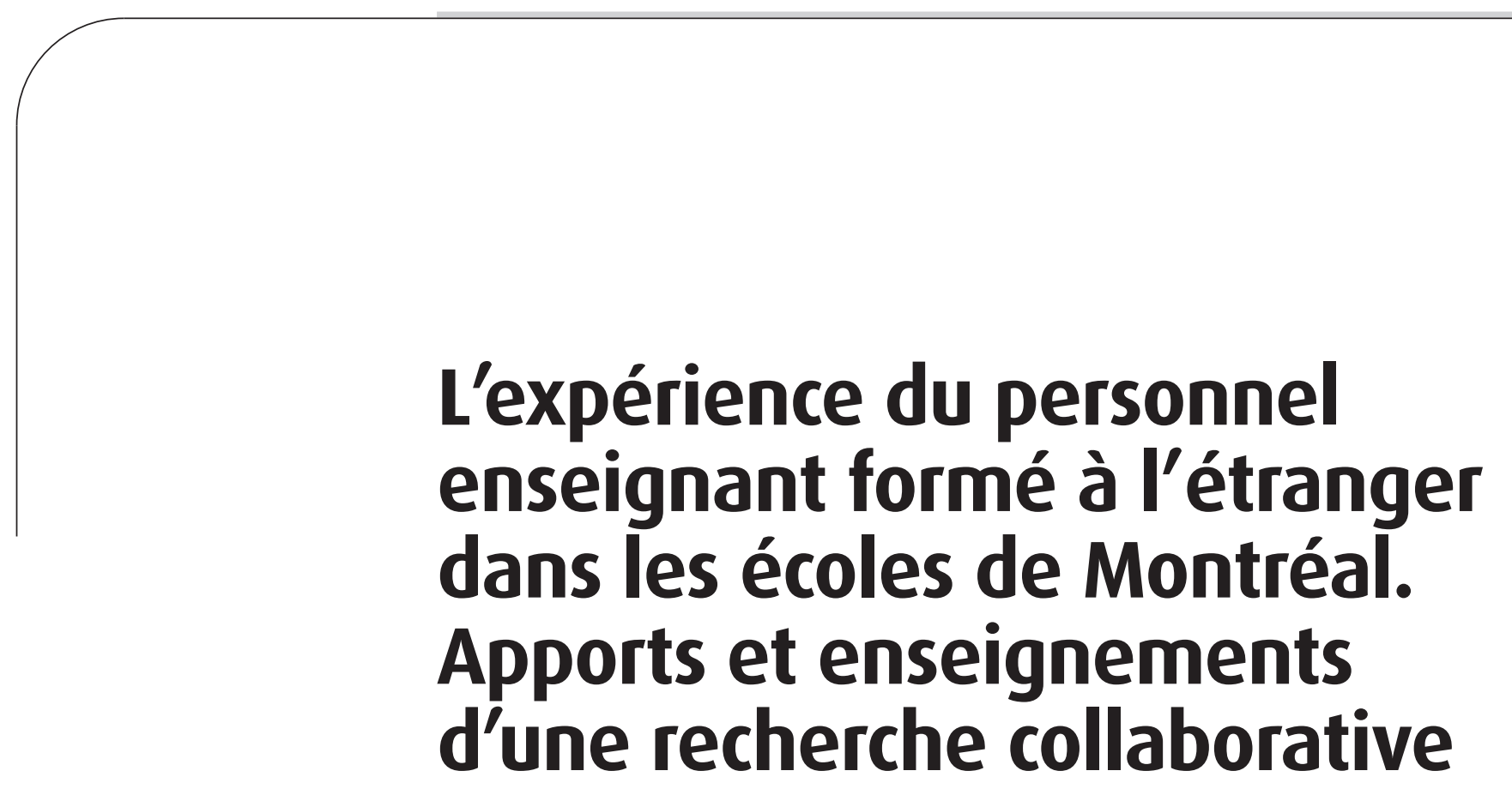

Citer cet article :

MORRISSETTE, J. et DEMAZIÈRE, D. (2018). L'expérience du personnel enseignant formé à l'étranger dans les écoles de Montréal. Apports et enseignements d'une recherche collaborative. Éducation et froncophonie, 46(2), 189-207.

Joëlle MORRISSETTE

Université de Montréal, Québec, Canada

Didier DEMAZIÈRE

Sciences Po, Paris, France

\section{RÉSUMÉ}

L'intégration professionnelle du personnel enseignant formé à l'étranger travaillant dans les écoles montréalaises est un enjeu d'importance. S'il suit une courte formation, c'est surtout au cœur des situations de travail qu'il réapprend le métier. Jusqu'à présent, la focale a surtout été mise sur les difficultés d'intégration des membres de ce personnel enseignant dans leur nouvel environnement de travail. À rebours, une approche interactionniste de l'intégration d'un groupe minoritaire à un groupe majoritaire conduit plutôt à considérer leurs expériences comme le produit de conventions professionnelles, propres aux contextes de travail, qui s'entrechoquent. La réalisation d'entretiens individuels et collectifs avec des enseignantes et des enseignants formés à l'étranger et avec certains de leurs partenaires a permis une coanalyse des événements marquants ayant jalonné leurs premières expériences dans les écoles montréalaises, et traduisant des tensions avec leurs expériences d'enseignement 
antérieures à la migration. Dans cette contribution, nous mettons l'accent sur les chocs entre conventions tacites définissant le travail enseignant et en examinons trois dimensions: les relations de travail, l'expertise au fondement du métier et les sources de la reconnaissance professionnelle. Enfin, nous discutons de l'intérêt du dispositif collaboratif pour les participantes et les participants et, au-delà, pour les personnels éducatifs, notamment les directions d'établissement qui accueillent du personnel enseignant de différents horizons dans leur équipe-école.

\section{ABSTRACT}

\section{The experience of internationally educated teachers in Montreal schools Contributions and lessons from a collaborative study}

Joëlle Morrissette, University of Montreal, Quebec, Canada

Didier Demaziere, Sciences Po, Paris, France

The professional integration of internationally educated teachers working in Montreal schools is an important issue. After taking a short course, they will be relearning the profession mostly in work situations. So far, the focus has been on the challengs of integrating members of this teaching staff into their new work environment. On the other hand, an interactionist approach to integrating a minority group into a majority group leads one to consider their experiences as the product of professional conventions specific to working contexts that collide. Conducting individual and collective interviews with internationally educated teachers and some of their partners allowed a co-analysis of the milestones that marked their initial experiences in Montreal schools, and reflected tensions in their teaching experiences prior to migration. In this contribution, we focus on collisions between tacit agreements that define the work of the teacher and examine three dimensions: labour relations, expertise at the foundation of the profession and sources of professional recognition. Finally, we discuss how well the collaborative process can work for participants and also for the educational staff, particularly school principals who host teachers from different backgrounds on their school team. 


\section{RESUMEN}

\section{La experiencia del personal docente formado en el extranjero en las escuelas de Montreal. Aportes y enseñanzas de una investigación colaborativa}

Joëlle MORRISSETTE, Universidad de Montreal, Quebec, Canadá

Didier DEMAZIÈRE, Ciencias políticas, Paris, Francia

La integración profesional del personal docente formado en el extranjero que trabaja en las escuelas de Montreal es un desafío importante. Aunque siguen una formación corta, es sobre todo en el seno de situaciones de trabajo en donde re-aprenden el oficio. Hasta ahora, se ha focalizado esencialmente las dificultades de integración de los miembros de personal docente en el nuevo entorno de trabajo. Al contrario, un planteamiento interaccionista de la integración de un grupo minoritario en un grupo mayoritario conduce más bien a considerar sus experiencias como el producto de convenciones profesionales, propias a los contextos de trabajo, que se entre-chocan. La realización de entrevistas individuales y colectivas con los maestros y maestras formadas en el extranjero y con algunos de sus colegas, ha facilitado el co-analisis de los hechos significativos que han marcado sus primeras experiencias en las escuelas montrealenses, y traducen las tensiones con sus experiencias de enseñanza anteriores a la migración. En la presente contribución, ponemos el acento sobre los choques entre convenciones tácitas que definen el trabajo docente y examinamos tres dimensiones: las relaciones de trabajo, la pericia con las bases del oficio y las fuentes de reconocimiento profesional. Finalmente, discutimos el interés de un dispositivo colaborativo para los participantes y, más aun, para el personal docente, sobre todo para las direcciones de escuelas que acogen en sus equipos al personal docente proveniente de diversos horizontes.

\section{INTRODUCTION}

Le Canada accueille de nombreux immigrantes et immigrants, notamment pour faire face à une pénurie de main-d'œuvre qualifiée, y compris dans le secteur de l'enseignement qui concerne près de $13 \%$ des 284080 des personnes immigrées recensées en 2006 (Zietsma, 2010). Dans la province de Québec, chaque année, 350 immigrantes et immigrants reçoivent du ministère de l'Éducation, du Loisir et du Sport (2013) un permis temporaire d'enseigner. Pour obtenir le brevet d'enseignement, soit 
l'autorisation permanente, ils doivent suivre 15 crédits de cours $^{1}$ et réussir un stage probatoire. Même si la plupart ont déjà enseigné avant de migrer, ils sont souvent confrontés à des attentes et à des normes nouvelles, ainsi qu'à des manières de faire qui tranchent avec leurs pratiques antérieures.

Dans le cadre d'une recherche portant sur la socialisation professionnelle d'enseignantes et d'enseignants formés à l'étranger dans les écoles montréalaises (Conseil de recherches en sciences humaines du Canada, 2015-2017), nous avons analysé leurs premières expériences au travail en prêtant attention aux événements qui constituent pour eux des chocs, et aux situations que certains de leurs partenaires de travail interprètent comme des faux pas. Notre contribution s'appuie sur une méthode interactionniste reposant principalement sur des entretiens collectifs inscrits dans une recherche collaborative réunissant du personnel enseignant formé à l'étranger et d'autres acteurs éducatifs. Ce dispositif de recherche-formation a permis aux participantes et aux participants de débattre des manières d'exercer le métier d'enseignant à partir de situations vécues. Favorisant l'explicitation des normes qui s'entrechoquent au cœur des événements coanalysés, ce dispositif, inscrit dans une démarche de recherche, a été approprié par les participants comme un espace de discussion et d'intercompréhension, suscitant une mise à distance critique.

\section{PROBLÉMATIQUE: L'INTÉGRATION DANS LES CONTEXTES DE TRAVAIL}

L'intégration professionnelle du personnel enseignant formé à l'étranger soulève de multiples enjeux de tous ordres (éducatifs, organisationnels, etc.) qui ont des traductions au cœur des situations concrètes de travail. Deux recensions ont porté sur l'accueil des enseignantes et des enseignants formés à l'étranger dans différents pays, dont le Canada, les États-Unis, l'Australie, le Royaume-Uni et Israël (Morrissette, Diédhiou et Charara, 2014; Niyubahwe, Mukamurera et Jutras, 2013). Elles montrent que trois questions majeures ont été traitées: celle du rôle des programmes universitaires visant à les préparer à leur travail dans les sociétés d'accueil (Cruickshank, 2004; Duchesne, 2010; Myles, Cheng et Wang, 2006; Ross, 2001; Walsh et Brigham, 2008), celle des conditions d'accès à l'emploi et des modes d'affectation dans les écoles (Cardu et Sanschagrin, 2002; Jabouin et Duchesne, 2012; Michael, 2006; Mujawamariya, 2008; Phillion, 2003; Schmidt, 2010) et celle de leur intégration au travail.

Ont ainsi été documentés deux types de difficultés rencontrées par les enseignantes et les enseignants formés à l'étranger. Sur le plan pédagogique, les écrits soulignent le caractère partiel de leur connaissance des curriculums et mettent en exergue des écarts entre les manières d'enseigner dans les pays d'accueil et d'origine, où la

1. Plus précisément, il s'agit de trois crédits sur le système scolaire québécois, au moins six sur la didactique, trois sur l'évaluation des apprentissages et trois autres sur l'intervention auprès des élèves handicapés ou en difficulté d'adaptation ou d'apprentissage. 
transmission magistrale de savoirs à mémoriser est souvent très répandue (Collin et Lévesque, 2013; Cruickshank, 2004; Myles et al., 2006; Peeler et Jane, 2005). Sur le plan relationnel, des tensions marquent les relations avec les élèves qui n'adoptent pas les conduites de déférence attendues par ces enseignantes et ces enseignants habitués à des rapports verticaux et autoritaires (Elbaz-Luwisch, 2004; Gordon, 1996; Hutchison, 2005). Les écrits signalent aussi des rapports problématiques avec leurs hiérarchies et leurs collègues: collaborations difficiles, dévaluation de leurs compétences en raison des écarts entre leurs pratiques et ce que l'on attend d'eux lors de la période probatoire (Bascia, 1996; Jabouin et Duchesne, 2012; Michael, 2006; Mujawamariya, 2008; Peeler et Jane, 2005; Remennick, 2002). Les parents d'élèves douteraient aussi parfois de leurs compétences et critiqueraient leur maîtrise de la langue officielle (Lefebvre, 2011; Phillion, 2003).

Privilégiant le point de vue d'enseignantes et d'enseignants formés à l'étranger, ces recherches mettent en lumière les embûches rencontrées. Cependant, l'idée de conformité sous-jacente mérite d'être examinée pour deux raisons. D'abord, parce qu'elle connote une perspective déficitaire, situant d'emblée les enseignantes et les enseignants formés à l'étranger "en défaut de», et négligeant le fait qu'ils ont des manières constituées, apprises et expérimentées d'exercer leur métier, fussent-elles différentes des exigences de leur nouveau milieu de travail. Ensuite, parce qu'elle est combinée à un prisme individualisant qui, dans la plupart des études, tend à négliger le rôle des partenaires de travail dans les processus d'intégration, une critique formulée aussi par Bertheleu (2012). Pour surmonter ces deux obstacles, la sociologie interactionniste offre de solides appuis théoriques.

\section{CADRE THÉORIQUE: UNE SOCIOLOGIE INTERACTIONNISTE}

L'interactionnisme d'Howard Becker $(1982,1986)$ invite à déplacer le regard de l'individu vers des réseaux d'interdépendance et des activités coordonnées. Il se situe à distance de la perspective dominante dans les sciences de l'éducation qui loge l'explication des conduites humaines «sous la peau et entre les oreilles» (Mehan, 1996) plutôt que dans les contextes dans lesquels elles se déploient.

Tout contexte d'action, depuis la salle de classe jusqu'au système éducatif dans son ensemble, est un espace social dans lequel est engagée une pluralité d'acteurs qui interagissent. Pour Becker (1982), toute activité professionnelle implique la coordination d'acteurs inscrits dans une division articulée du travail et participant à des réseaux coopératifs ou concurrentiels. En somme, tout travail est inscrit dans un «monde social» (Becker, 1982) composé d'acteurs ayant des rôles spécifiques et dont l'interdépendance coalise une écologie professionnelle (ex.: personnel enseignant, directions d'établissement, parents, personnel administratif, élèves, etc.). Dans de tels mondes, les interactions sont réglées par des routines et des normes qui associent les acteurs impliqués dans la production d'un travail (ici, l'enseignement), 
et qui sont le vecteur de réponses réciproques prévisibles et régularisées. Ces mondes s'appuient sur des «conventions» ou des «compréhensions partagées», soit des accords mutuels qui facilitent la coopération et la coordination des activités, et qui rendent possible le travail. Ces savoir-faire partagés sont réifiés en routines et en normes (tacites), définissant les procédures à suivre, les manières de faire, les conduites pertinentes et déviantes, et les relations réciproques entre acteurs. Toutefois, ces conventions autorisent des marges de jeu, et elles peuvent être ajustées et renégociées dans le flux des interactions quotidiennes.

Cette sociologie interactionniste invite à voir les enseignantes et les enseignants formés à l'étranger comme des membres d'un monde professionnel dont ils ignorent les conventions - au moins en partie - et dans lequel ils importent d'autres conventions intériorisées dans un autre système éducatif. Ils risquent dès lors d'être perçus comme des déviants ou des outsiders (Becker, 1963/1985) qui ne respectent pas les normes tacites. Cela conduit à reformuler la question de leur intégration professionnelle: ils sont des outsiders qui doivent s'inscrire dans un groupe majoritaire, mais ils ont aussi accumulé des expériences articulées à des conventions du travail distinctes, relatives à un autre contexte. Cela invite à envisager que les difficultés qu'ils affrontent sont moins des traces d'un déficit de savoir-faire que des signes de chocs entre conventions professionnelles différentes.

\section{MÉTHODOLOGIE: UNE RECHERCHE COLLABORATIVE COMME ESPACE D'EXPLICITATION ET D'INTERCOMPRÉHENSION}

Nous avons déployé une recherche collaborative (Conseil de recherches en sciences humaines, 2015-2017) impliquant quatre enseignantes et enseignants formés à l'étranger et travaillant au secondaire. L'objectif de cette recherche-formation était d'engager l'équipe de recherche et les professionnels dans une démarche de coproduction de savoirs inédits et négociés (Desgagné, 2001; Morrissette, Mottier Lopez et Tessaro, 2012). La recherche s'articule donc à une visée de soutien à l'intégration professionnelle pour ces membres du personnel enseignant formés à l'étranger, le dispositif étant destiné à favoriser une prise de recul, voire une distanciation critique par rapport à leurs premières expériences au Québec. Les quatre membres du corps enseignant étaient issus d'Europe de l'Est (e1) et d'Afrique du Nord (e2, e3, e4), et ils travaillaient tous dans une même commission scolaire située sur l'île de Montréal (cf. Tableau 1). 
L'expérience du personnel enseignant formé à l'étranger dans les écoles de Montréal.

Apports et enseignements d'une recherche collaborative

Tableau 1. Profils des enseignantes et des enseignants formés à l'étranger

\begin{tabular}{|c|l|l|l|l|}
\hline \multicolumn{1}{|c|}{} & $\begin{array}{l}\text { Pays } \\
\text { d'origine }\end{array}$ & $\begin{array}{l}\text { Formation } \\
\text { initiale }\end{array}$ & $\begin{array}{l}\text { Expérience } \\
\text { dans le pays } \\
\text { d'origine }\end{array}$ & Contexte de leur première année au Québec \\
\hline e2 & Moldavie & $\begin{array}{l}\text { Licence en } \\
\text { anglais, langue } \\
\text { étrangère }\end{array}$ & 3 ans & $\begin{array}{l}\text { Immigre en 2007. Obtient un diplôme en enseignement au Québec en 2010. } \\
\text { Décroche trois petits contrats en enseignement de l'anglais dans trois écoles } \\
\text { différentes de Montréal (64 \% d'une tâche). }\end{array}$ \\
\hline e3 & Algérie & $\begin{array}{l}\text { Licence en } \\
\text { mathématiques }\end{array}$ & 13 ans & $\begin{array}{l}\text { Immigre en 2010. Premier contrat dans son domaine dans une école privée } \\
\text { français, langue } \\
\text { secondaire de Montréal. Suit les 15 crédits de cours obligatoires et trois stages. }\end{array}$ \\
\hline e4 & Tunésie & $\begin{array}{l}\text { Maîtrise en } \\
\text { sciences } \\
\text { naturelles }\end{array}$ & 16 ans & $\begin{array}{l}\text { Immigre en 2012. Suit les 15 crédits de cours obligatoires, mais sans stage. } \\
\text { Décroche un contrat de neuf mois dans une école publique secondaire de } \\
\text { Montréal. }\end{array}$ \\
\hline
\end{tabular}

À l'automne 2016, ils ont d'abord participé à un entretien biographique (Demazière, 2011) portant sur leur activité enseignante dans leur pays d'origine, leur parcours migratoire et leurs premières expériences dans les écoles montréalaises. Ces entretiens ont permis de saisir les référentiels professionnels auxquels ils ont été socialisés dans leur pays d'origine. Ils ont mis en relief de fortes convergences entre eux, à l'exception d'un aspect: si l'idéologie politique socialiste percolait dans le travail de l'enseignant originaire d'Europe orientale, pour les trois autres, c'était davantage l'idéologie religieuse qui obligeait à afficher de grandes qualités «morales», notamment dans une distance à maintenir pour les enseignantes travaillant auprès de jeunes garçons.

Ces entretiens étaient préparatoires à cinq entretiens collectifs (EG1, EG2, etc.) impliquant ces mêmes enseignantes et enseignants, auxquels ont été ajoutés d'autres acteurs: en EG3, un représentant d'un organisme œuvrant à l'insertion professionnelle des enseignantes et des enseignants (ro); en EG4, un conseiller pédagogique de la commission scolaire dans laquelle travaillent les quatre enseignantes et enseignants formés à l'étranger (cp); en EG5, une direction d'établissement (dé) et un parent d'élèves (pé). Les questions posées en situation d'entretien étaient axées sur les expériences d'enseignement au Québec des membres du personnel enseignant formés à l'étranger, sur les déboires et les incidents rencontrés, ainsi que sur les ajustements apportés à leurs pratiques habituelles. Le choix d'impliquer d'autres acteurs constitue l'un des principaux atouts du dispositif: leurs réactions ont permis d'enrichir l'interprétation des turbulences évoquées par les enseignantes et les enseignants formés à l'étranger, d'expliciter les cadrages normatifs sous-jacents et les façons de les négocier dans les écoles montréalaises. L'autre atout a été la traduction de la ficelle méthodologique de la comparaison de cas de Becker (2013), mobilisée en situation d'entretien. En comparant leurs expériences dans leur pays d'origine à celles dans les 
écoles montréalaises, nous avons pu rendre manifeste la confrontation des systèmes de conventions tacites, laquelle pose un problème lors de leur intégration, ce qui a stimulé la réflexivité de tous les participants. Nous y revenons dans la discussion.

Les analyses présentées ici reposent principalement sur les entretiens collectifs, moments d'échange sur ce qui constituait pour les uns des chocs et pour les autres des faux pas par rapport aux habitudes locales. Une analyse de conversations (Morrissette, 2011) a permis de faire apparaître des conventions distinctes adossées à des expériences spécifiques, qui différencient les membres du personnel enseignant formés à l'étranger de leurs partenaires. Nous renseignons ici les écarts dans les manières de concevoir le travail et de pratiquer le métier d'enseignant en privilégiant trois dimensions, centrales pour l'activité: le statut de l'enseignante ou de l'enseignant dans les relations de travail, l'expertise au fondement de son rôle professionnel et les bases de la reconnaissance professionnelle.

\section{RÉSULTATS: LA CONFRONTATION DE SYSTÈMES DISTINCTS DE CONVENTIONS TACITES}

Les enseignantes et les enseignants formés à l'étranger se heurtent à des conventions professionnelles auxquelles ils ne sont pas préparés et qu'ils peinent à comprendre. Nous en examinons ici trois dimensions qui sont apparues structurantes pour caractériser le métier d'enseignant. Pour chacune, nous expliquons comment les conventions assimilées dans le système éducatif du pays d'origine sont révélées à travers des pratiques qui s'avèrent non viables dans les écoles montréalaises, et comment les réactions - conseils, protestations, etc. - de partenaires de travail sont des traductions de conventions «locales».

\section{Le statut dans les relations de travail}

Le statut du personnel enseignant dans les relations de travail est configuré de manière différenciée au Québec et dans les pays d'origine des enseignantes et des enseignants formés à l'étranger: acquis et donné ici, conquis et négocié là-bas. Les relations avec les partenaires de travail sont bien différentes, et les discussions au sein de notre dispositif contribuent à révéler ces écarts et à discuter des façons de les combler. Dans leur pays d'origine, ces enseignantes et ces enseignants bénéficiaient d'un statut garanti par la formation initiale et souligné par des marqueurs matériels (estrade en classe, port d'une blouse), situant les élèves (et les parents) dans une distance déférente. Tous les quatre disent avoir été investis d'une autorité forte et crainte, de sorte que leur travail leur semblait facilité en comparaison avec le contexte d'arrivée: «Dans notre pays, nous avons juste une mission; tu entres dans la classe et tu enseignes; c'est tout.» (EG4 e1 471-472) 
La pratique du métier s'inscrivait dans un cadrage ferme des rapports avec les élèves, autour du couple autorité/obéissance. Cela n'exclut pas l'indiscipline, mais celle-ci était gérée par un surcroît de sévérité. La transposition de cette expérience en contexte montréalais provoque de multiples tensions, générées par des conduites anodines, comme le rapporte un des enseignants à propos d'un regard perçu comme un marqueur d'intimidation par un élève:

«Pourquoi vous me regardez?» C'est ce qui m'est arrivé cette semaine avec un élève vraiment difficile; je n'ai pas dit «tu déranges» ou quelque chose comme ça; je l'ai juste regardé dans les yeux; [...] et j'ai dit: «Parce que j'ai mes yeux et j'ai le droit de te regarder»; il m'a dit: «Vous m'intimidez si vous me regardez comme ça»; [...] le mot «intimidation» semble être le premier mot qu'un élève d'ici commence à dire. (EG2 e1 700-713)

Reproduire une attitude autoritaire - ou perçue comme telle - provoque des turbulences si importantes qu'il devient impossible d'enseigner: les quatre enseignantes et enseignants affirment que lorsqu'ils adoptent cette attitude, les élèves deviennent vite dissipés, se montrent impolis, refusent de faire les tâches, etc. En rapportant une intervention de soutien auprès d'un enseignant formé à l'étranger, l'un des partenaires aide à comprendre comment reprendre un élève selon les normes «locales", et plus globalement comment susciter l'adhésion des élèves en adoptant d'autres attitudes:

Je rencontre Marie et dis: «Comment vous allez créer votre lien avec les élèves? Qu'est-ce qui vous fait peur?» [...] Elle me dit: «Ma crainte, c'est mon accent et des mots mathématiques que j'ai l'impression de ne pas saisir» [...] Alors on a travaillé, elle est rentrée dans la classe en disant aux élèves: «Je sais que j'ai un accent et on va s'habituer»; au lieu que ce soit les élèves qui en parlent, elle en a parlé elle-même; et elle a dit: «Vous allez m'aider [...] à apprendre mieux mes termes mathématiques et mon langage québécois.» Et c'est comme ça qu'elle les a eus [...] Alors ils étaient devenus vraiment des alliés et ça a très bien fonctionné [...] Je lui ai dit: "Ça serait intéressant que tu présentes qui tu es»; elle a fait un diaporama pour présenter d'où elle venait; comment ça se passait dans son pays. (ro, EG3 592-614)

Devant ce qu'ils décodent comme de l'indiscipline, les enseignantes et les enseignants interrogés ont des réponses décalées, par exemple en excluant de la classe les élèves les plus turbulents. Formuler cette routine de travail permet aux autres acteurs présents lors des trois derniers entretiens de comprendre le fondement de réactions jugées inappropriées. Cette direction d'établissement peut alors expliciter les manières de faire requises pour affronter l'indiscipline: 
Avant les interventions ciblées qui nécessitent des ressources accrues, il y a quelque chose qui appartient tout d'abord à la salle de classe, à l'enseignant [...] et parfois on va lui dire «là je te renvoie [à ta classe] et j'aimerais que tu essaies une solution avant qu'on fasse entrer le technicien en éducation spécialisée» [...] il faut prendre l'élève à part, l'amener dans le corridor, lui parler, lui mettre un message à côté de lui discrètement pour lui dire «là, tu te calmes parce que tu déranges les autres »; tu sais, il y a plusieurs stratégies pour un élève qui parle dans la classe [...] on tente des choses avant de le sortir. (EG5 461-649)

Les parents d'élèves contribuent aussi à diffuser les normes pratiques en vigueur en intervenant directement dans les écoles montréalaises où travaillent les quatre enseignantes et enseignants formés à l'étranger. Cela tranche avec la distance entre les parents et la communauté éducative qu'ils ont connue précédemment: «Quand l'enfant est scolarisé [...], il est coupé de la maison, les parents n'ont rien à faire ou à dire dans le milieu scolaire» (EG5 e2 306-309). Ces informations livrées en entretien permettent aux partenaires de travail de comprendre pourquoi les interventions des parents suscitent des résistances ou des incompréhensions de la part de ces enseignantes et enseignants formés à l'étranger. Un parent d'élève raconte comment il est intervenu auprès de l'enseignant de son enfant:

Je suis allée le voir et là il pensait que je remettais en cause ses diplômes; j'ai dit: «Non, je ne remets pas en cause tes diplômes; c'est juste l'approche qui n’est pas nécessairement la meilleure» [...] Il m’a dit: «Moi, je suis habitué à avoir des classes où les élèves se tiennent» [...] En arrivant ici, c'était le jour et la nuit. (EG5 121-204)

Pendant les entretiens, des conventions tacites, relatives ici aux relations de travail et à la position qu'y occupe le personnel enseignant, sont explicitées et discutées. Ces nouvelles compréhensions consolident l'intégration professionnelle des quatre membres du personnel enseignant formés à l'étranger et permettent aussi aux partenaires de travail de prendre la mesure des changements qui leur sont nécessaires pour enseigner dans les classes à Montréal. Des mécanismes similaires opèrent pour la deuxième dimension analysée.

\section{L'expertise au fondement du rôle professionnel}

Pour les enseignantes et les enseignants formés à l'étranger qui ont participé à l'enquête, la maîtrise des savoirs disciplinaires est le cœur de leur métier. Dans leurs pays d'origine, cette expertise est d'autant plus centrale que les contextes de travail sont dépourvus de ressources technologiques et marqués par des classes aux effectifs pléthoriques. Dès lors, l'enseignement magistral centré sur les notions théoriques à transmettre à un groupe-classe indifférencié apparaît comme la seule façon de faire. 
Cette définition du métier ne les prépare pas aux exigences des écoles montréalaises. Ils y expérimentent qu'elle est inopérante: les quatre enseignantes et enseignants formés à l'étranger rapportent que pendant leurs exposés, les élèves manifestent leurs incompréhensions, réclament des explications supplémentaires, finissent par décrocher et par perturber la classe. Ils découvrent aussi que l'apprentissage par cœur est proscrit, puisque les élèves disposent de leurs notes de cours lors des examens, ce qu'ils éprouvent comme une remise en cause de leur expertise: «Ma fonction d'enseignant n'existe plus; c'est plus la fonction de gardienne» (EG5 e2 885). Un conseiller pédagogique explique alors: «Dans le programme, maintenant on privilégie des tâches un peu plus complexes dans lesquelles les élèves n'ont pas nécessairement à tout retenir [...] il faut les habituer à résoudre des problèmes plus complexes» (EG4 219-222).

Lors des entretiens, les partenaires professionnels énoncent une convention partagée: pas d'apprentissage sans l'instauration préalable d'une relation enseignant-élèves favorisant la participation de ceux-ci. C'est ce que souligne l'un d'eux: "C'est ma $34^{\mathrm{e}}$ année [...] et partout j'ai vu que si on n'a pas créé le lien avec les élèves, même si on a le meilleur contenu disciplinaire du monde entier, ça ne fonctionnera pas» (ro, EG3 835-843). Construire une relation avec les élèves est une composante importante de la profession enseignante au Québec, comme l'exprime une enseignante formée à l'étranger au cours des discussions: «[En Algérie, le rapport à l'élève est] rigide; c'est ça qui est différent; ici, c'est la relation POUR transmettre; donc c'est plus le côté humain» (EG4 e2 842-843).

Une autre dimension de l'expertise, saillante dans les écoles montréalaises caractérisées par une forte hétérogénéité, est la capacité à différencier son enseignement. Les adaptations que cela implique pour les quatre membres du personnel enseignant formés à l'étranger sont parfois réclamées par les parents: inquiète des performances de sa fille, cette mère alerte en vain son enseignant, puis sollicite une collègue de celui-ci afin qu'elle l'assiste: «L'autre enseignante me disait: “J'essaie de donner des choses, mais il ne veut pas les avoir; dans le fond, il veut faire SES choses" (EG5 pé 143-151). La narration de cet épisode conduit l'un des enseignants formés à l'étranger à fournir une explication à la résistance de cet enseignant, qui semble avoir du sens en référence aux expériences antérieures des trois autres participants, révélant ainsi une conception tacite de l'expertise et de la compétence:

Tout ce qui est autisme, ce qui est difficulté d'apprentissage, ce qui est relatif aux besoins spécifiques, on n'a pas été formés pour cela; pour nous, c'est vraiment l'académique; et la collègue qui vous a dit «j'essaierai de lui donner du matériel, de lui donner des planifications", je comprends l'enseignant parce que moi, si tu viens me dire «voilà, je te donne la planification du cours; tu fais les exercices comme ceci», c'est comme si tu remets ma compétence d'enseignant en cause. (EG5 e3 362-369) 
Ces propos informent les partenaires de travail que les enseignantes et les enseignants formés à l'étranger participant à la recherche ne sont guère préparés à prendre en compte les difficultés d'apprentissage, encore moins celles liées à diverses problématiques de développement. C'est que, dans leur pays d'origine, les élèves concernés sont stigmatisés et traités à part, considérés comme "paresseux» ou «handicapés» et placés dans des institutions spécialisées (EG2 e2 1119). Dans les écoles montréalaises, l'accompagnement des orthopédagogues et des éducateurs spécialisés, qui expliquent le sens des étiquettes (autisme, dyslexie, etc.) ou les manières de construire l'inclusion en classe, fait évoluer les pratiques des enseignantes et des enseignants formés à l'étranger. Mais le grand écart initial peut expliquer que certains d'entre eux adoptent des attitudes perçues par leurs partenaires comme rigides ou incompréhensibles, comme le rapporte la direction d'établissement:

Je me souviens d'un cas où il y avait un autiste intégré [...] il m'avait dit «c'est la maladie mentale!» [...] je dis «non, ce n'est pas une maladie mentale; c'est une condition ça»; «je ne peux pas croire que les parents des autres enfants acceptent ça; [... moi] je ne l'accepterais pas»; donc, on était vraiment dans des conceptions très ancrées. (EG5 1081-1112)

À propos de l'expertise au cœur de la définition du métier, les discussions lors des entretiens collectifs améliorent l'intercompréhension des exigences propres au contexte montréalais comme des résistances issues de l'intériorisation d'autres conventions de travail. Des observations comparables peuvent être faites dans la troisième dimension étudiée.

\section{Les sources de la reconnaissance professionnelle}

La définition de ce qu'est un bon enseignant ou une bonne enseignante renvoie aussi à des conventions tacites au principe de la reconnaissance professionnelle. Le dispositif montre combien celle-ci est adossée à des règles dissemblables entre les pays d'origine des quatre enseignantes et enseignants formés à l'étranger et le Québec. Dès lors, le passage d'un monde professionnel à l'autre implique bien plus qu'une adaptation: il exige une conversion.

Un des plus grands chocs pointés par les membres du personnel enseignant formés à l'étranger, et un faux pas majeur évoqué par leurs partenaires, concerne l'évaluation des apprentissages. Celle-ci vise à identifier et à sélectionner les élèves les plus performants dans les systèmes scolaires élitistes dans lesquels ces quatre enseignantes et enseignants ont débuté (EG4 e3 174-180). Transposée aux écoles montréalaises, cette perspective se heurte aux objectifs officiels de réussite et de progression de tous les élèves, et elle soulève les protestations des élèves et de leurs parents, conduisant parfois à solliciter les conseils de pairs: 
Je lui montrais les notes des évaluations pour savoir s'il y avait trop d'élèves en échec; il m’a dit: «s'il y a trop d'élèves en échec, ça ne marche plus, il faut que tu revoies un peu tes évaluations» [...] donc, il essayait de me guider un tout petit peu pour que je n'aie pas de problème. (EG2 e4 134-141)

Quoi qu'il en soit, l'adoption de nouvelles pratiques évaluatives est rapide, tant la pression à l'alignement est forte. Avec le temps, les principes de l'évaluation critériée en vigueur au Québec, qui supposent de valoriser différents aspects de la tâche et de considérer les progressions des élèves, sont mieux compris et suscitent l'adhésion, comme l'exprime l'enseignant intégré depuis plus longtemps que les trois autres:

[Au Québec,] on attend de l'élève qu'il puisse traiter cet exercice, mais sans forcément trouver la bonne réponse [...] il ne sera pas mal évalué parce qu'il n'a pu traiter le problème; donc, les évaluations, ce n'est pas la même chose; nous, on a l'échec automatiquement; si dès le départ il s'est trompé, on peut l'excuser une fois, sur une petite erreur de calcul; s'il refait une autre erreur de calcul, c'est fini: il est en échec [...] par contre, ici, c'est plutôt l'évolution. (EG2 e2 836-844)

Comme le racontent les quatre enseignantes et enseignants formés à l'étranger, livrant ainsi des clés de compréhension à leurs partenaires, l'évaluation élitiste et sélective est étroitement associée à la reconnaissance professionnelle dans leurs pays d'origine. En effet, la réussite des élèves est directement attribuée au travail des membres du corps enseignant, qui reçoivent d'autant plus de gratifications que leurs élèves sont performants.

Chaque année, dans notre pays, au printemps, il y a des olympiades pour chaque matière [...] l'enseignant qui réussit à envoyer le plus d'élèves qui gagnent des prix de première place est toujours... [...] moi, j'étais connue comme élève, mais mon enseignante qui m’a envoyée était plus connue encore pour ça. (EG4 e1 1064-1073)

Cette reconnaissance symbolique favorise aussi la progression de carrière et permet d'obtenir des classes plus avancées dans lesquelles les élèves ont été triés, puisque «tu peux arriver dans une classe en terminale si tu as le quota en taux de réussite» (EG4 e3 1031-1032). Elle peut aussi permettre d'être distingué par les inspecteurs pour délivrer des prestations à des pairs (EG4 e2 770-771), et elle attire les parents d'élèves qui les sollicitent pour donner des cours privés dans les quatre pays d'origine:

Premièrement, les parents veulent justement que leur enfant soit dans la classe de cet enseignant; deuxièmement, [... il] est sollicité pour des cours particuliers après l'école [...], donc l'enseignant qui a ce statut-là bénéficie d'une source financière supplémentaire. (EG4 e1 701-707) 
Le modèle professionnel, tacite et mis au jour par notre dispositif, apparaît contradictoire avec la convention délimitant ce qu'est un bon enseignant ou une bonne enseignante dans le contexte québécois. Au-delà de l'évaluation des élèves, c'est le rapport à l'activité qui est en jeu. À l'opposé du sentiment de pleine maîtrise de son travail, il s'agit de chercher l'amélioration de sa pratique au contact de ses partenaires de travail. Conformément au modèle intériorisé initialement, les quatre enseignantes et enseignants formés à l'étranger disent avoir tendance à dissimuler les difficultés rencontrées dans le nouveau contexte de travail: «Si tu demandes les services d'une conseillère pédagogique, c'est parce qu'on te considère comme "nul", comme quelqu'un qui ne sait pas faire sa job» (EG3 e3 713-715). En réponse, les partenaires de travail explicitent une autre norme: au Québec, ne pas partager ses difficultés en vue de définir un plan d'action serait interprété comme un manque de professionnalisme. Au contraire, le professionnel cherche constamment à se perfectionner en dialoguant avec ses pairs ou sa direction, en suivant des formations, en manifestant son implication réflexive et son intégration active à l'échelle de l'école.

Ainsi, les entretiens collectifs permettent aux participantes et aux participants de saisir l'ampleur des écarts entre les conventions implicites qui encadrent la reconnaissance professionnelle: dans un cas, elle se mesure par la carrière ascendante au sein du groupe professionnel et par la capacité individuelle à produire des performances scolaires; dans l'autre, elle se joue au sein des collectifs de travail à l'intérieur des écoles et par l'aptitude à enrichir sa pratique afin de faire face à des situations hétérogènes.

\section{DISCUSSION CONCLUSIVE}

Notre dispositif collaboratif a produit une intercompréhension réciproque, que les participantes et les participants se sont appropriée, et qui sert le développement professionnel. Ainsi, la direction d'établissement nous a dit à maintes reprises avoir modifié ses manières de pratiquer la supervision pédagogique des enseignantes et des enseignants formés à l'étranger pendant leur probation: elle prend appui sur leurs expériences antérieures, liées au pays spécifique d'où ils viennent, et établit des comparaisons avec le contexte québécois afin de les amener à comprendre les fondements des conventions valides dans leur nouveau contexte de travail. Du côté des enseignantes et des enseignants formés à l'étranger, des effets positifs sont aussi documentés. Dans les entretiens biographiques, ils ont verbalisé les normes professionnelles intériorisées et devenues implicites, ce qui a favorisé un recul réflexif d'avec le système éducatif dans lequel ils travaillaient. Les nombreuses discussions lors des entretiens collectifs, entre eux et avec leurs partenaires de travail, ont favorisé la circulation et le partage de récits d'expériences et d'interprétation des situations relatées. Leurs normes professionnelles et leurs conceptions du métier ont ainsi pu évoluer et se rapprocher des valorisations opératoires dans les écoles où ils travaillent désormais. Par exemple, l'enseignant formé à l'étranger qui dépréciait la fonction 
enseignante au Québec en l'associant à du gardiennage s'est approprié les orientations concernant la perspective inclusive, au point d'y puiser un sens au métier: "Je ne me vois pas partir ailleurs» (e2 EG5 1577). Autre exemple: les quatre enseignantes et enseignants formés à l'étranger avancent que, depuis leur participation à la recherche collaborative, ils n'hésitent plus à s'inscrire dans les formations offertes par la commission scolaire, ce qui leur apparaissait auparavant comme un signe d'incompétence.

Au-delà de ces bénéfices directs, notre démarche collaborative pointe vers des besoins de formation. Plusieurs propositions ont été formulées pour améliorer l'intégration dans les écoles des élèves issus de l'immigration (ex. : Mc Andrew, Garnett, Ledent, Ungerleider, Adumati-Trache et Ait-Said, 2008). Mais l'intégration du personnel enseignant formé à l'étranger est tout aussi cruciale, dans un tel contexte de pénurie (Cloutier, 2018), que l'embauche accrue d'enseignantes et d'enseignants formés à l'étranger, qui est considérée comme la seule sortie de crise possible ${ }^{2}$. Nos résultats pourraient être mobilisés pour sensibiliser les personnels administratifs des commissions scolaires. Pensons en particulier aux entrevues de sélection passées par les enseignantes et les enseignants formés à l'étranger pour accéder aux listes de rappel. À l'heure actuelle, on leur propose des mises en situation, surtout relatives à la gestion de classe, afin d'évaluer leurs capacités à garder le contrôle sur les élèves, sans faire appel à des ressources spécialisées. Or, ces mises en situation leur sont complètement étrangères, surtout quand ils ont connu des rapports de places hiérarchiques qui imposent l'obéissance des élèves. Ils peinent ainsi à se mettre en valeur. Les membres des comités d'entrevue pourraient mettre l'accent sur leurs apports potentiels, avoir une ouverture pour leur savoir-faire forgé dans un autre contexte, potentiellement transférable dans les écoles montréalaises.

Nos résultats incitent aussi à des activités de sensibilisation et de formation d'autres personnels scolaires, notamment des directions d'établissement. Notre recherche montre que la (re)socialisation professionnelle des enseignantes et des enseignants formés à l'étranger procède souvent "par claques" (Zolesio, 2013) au cœur des situations de travail, par reproches, rappels à l'ordre et sanctions. Cela engendre des stratégies d'évitement chez ce personnel enseignant qui, sous probation, est fragilisé (Morrissette et Demazière, 2018), et cela contribue aussi à reconduire des incompréhensions de part et d'autre. Former les directions à l'organisation d'échanges d'expériences dans leur établissement permettrait de mettre en dialogue différentes cultures de travail, de concevoir les pratiques des nouveaux collègues formés à l'étranger comme des traces d'expériences acquises ailleurs, et non comme des «erreurs", comme des routines de travail qui, pour eux, fonctionnent comme allant de soi. Ainsi, les directions faciliteraient pour les uns la redéfinition - parfois radicale - de la valeur professionnelle engendrée par la migration géographique et, pour les

2. Communication personnelle du 15 juin 2018 avec la direction des ressources humaines de la commission scolaire dans laquelle travaillent les enseignantes et les enseignants formés à l'étranger qui ont participé à nos travaux. 
autres, la reconnaissance de savoir-faire qui ne sont pas définis à partir des standards occidentaux. Les directions devraient être formées à une approche compréhensive, puis former leur équipe à celle-ci pour accueillir une multiplicité d'expériences et de valeurs, et pour mobiliser les apports des enseignantes et des enseignants formés à l'étranger pour la culture de travail dans les écoles montréalaises. Ces enseignantes et ces enseignants ont notamment des manières d'intervenir auprès des élèves qui viennent d'immigrer, et ils ont une compréhension des attentes de leurs parents visà-vis de l'école qui peut être mise à profit.

L'intégration du personnel enseignant formé à l'étranger doit se faire au travers d'une ouverture réciproque, et non d'un processus d'assimilation.

\section{Références bibliographiques}

BASCIA, N. (1996). Inside and outside: Minority immigrant teachers in Canadian schools. International Journal of Qualitative Studies in Education, 9(2), 151-165. Repéré à https://www.tandfonline.com/doi/abs/10.1080/0951839960090204

BECKER, H. S. (1963/1985). Outsiders. Études de sociologie de la déviance. Paris, France: Métailié.

BECKER, H. S. (1982). Les mondes de l'art. Paris, France: Flammarion.

BECKER, H. S. (1986). Doing things together. Evanston, IL: Northwestern University Press.

BECKER, H. S. (2013). What about Mozart? What about murder. Chicago, IL: University of Chicago Press.

BERTHELEU, H. (2012). Discriminations et relations interethniques: quelques remarques. Diversité. Ville-École-Intégration, (168), 31-36.

CARDU, H. et SANSCHAGRIN, M. (2002). Les femmes et la migration: les représentations identitaires et les stratégies devant les obstacles à l'insertion socioprofessionnelle à Québec. Recherches féministes, 15(2), 87-122.

CLOUTIER, P. (2018, 12 mars). Une pénurie d'enseignants qui s'aggrave Le Soleil. Repéré à https://www.lesoleil.com/actualite/education/une-penurie-denseignantsqui-saggrave-c966ce80ff7008a644523e2f1b631b3f 
COLLIN, S. et LÉVESQUE, M. (2013). Enseignement et diversité culturelle 2: présentation. Québec français, (168), 47.

CRUICKSHANK, K. (2004). Toward diversity in teacher education: Teacher preparation of immigrant teachers. European Journal of Teacher Education, 27(2), 125-138.

DEMAZIÈRE, D. (2011). L'entretien biographique et la saisie des interactions avec autrui. Recherches qualitatives, 30(1), 61-83. Repéré à https://hal.archives-ouvertes. fr/hal-01510177/document

DESGAGNÉ, S. (2001). La recherche collaborative: nouvelle dynamique de recherche en éducation. Dans M. Anadón et M. L’Hostie (dir.), Nouvelles dynamiques de recherche en éducation (p. 51-76). Québec, Québec: Presses de l'Université Laval.

DUCHESNE, C. (2010). À propos de l'accompagnement avant et pendant les stages d'étudiants immigrants inscrits à un programme de formation à l'enseignement. Revue des sciences de l'éducation, 36(1), 95-115.

ELBAZ-LUWISCH, F. (2004). Immigrant teachers: Stories of self and place. International Journal of Qualitative Studies in Education, 17(3), 387-414.

GORDON, J. A. (1996). Teachers from different shores. Equity and Excellence in Education, 29(3), 28-36.

HUTCHISON, C. B. (2005). Teaching in America:A cross-cultural guide for international teachers and their employers. Dordrecht, Pays-Bas: Springer.

JABOUIN, S. et DUCHESNE, C. (2012). Le difficile parcours d'insertion des enseignants issus de l'immigration dans la profession enseignante en Ontario. Revue d'éducation de l'Université d'Ottawa, 2(1), 4-5.

LEFEBVRE, M. L. (2011). L'expérience d'enseignants de groupes minoritaires en milieu scolaire montréalais. Dans J. Tondreau et R. Marcel (dir.), L'école québécoise: débats, enjeux et pratiques sociales (p. 320-329). Montréal, Québec: CEC.

Mc ANDREW, M., GARNETT, B., LEDENT, J., UNGERLEIDER, C. ADUMATI-TRACHE, M. et AIT-SAID, R. (2008). La réussite scolaire des élèves issus de l'immigration: une question de classe sociale, de langue ou de culture? Éducation et francophonie, 36(1),177-196. Repéréàhttps://www.erudit.org/en/journals/ef/2008-v36-n1-ef2292 /018096ar.pdf

MEHAN, H. (1996). Beneath the skin and between the ears: A case study in the politics of representation. Dans S. Chaiklin et J. Lave (dir.), Understanding practice: Perspective on activity and context (p. 241-268). New York, NY: Cambridge University Press. 
MICHAEL, O. (2006). Multiculturalism in schools: The professional absorption of immigrant teachers from the former USSR into the education system in Israel. Teaching and Teacher Education, 22(2), 164-178. Repéré à https://www. sciencedirect.com/science/article/pii/s0742051X05001265

Ministère de l'Éducation, du Loisir et du Sport. (2013). Rapport d'évaluation. Politique d'intégration scolaire et d'éducation interculturelle. Québec, Québec: Gouvernement du Québec.

MORRISSETTE, J. (2011). Vers un cadre d'analyse interactionniste des pratiques professionnelles. Recherches qualitatives, 30(1), 38-59. Repéré à http://www. recherche-qualitative.qc.ca/documents/files/revue/edition_reguliere/numero30(1)/ RQ_30(1)_Morrissette.pdf

MORRISSETTE, J. et DEMAZIÈRE, J. (2018). Dualité des processus de socialisation professionnelle des enseignants formés hors Québec: entre imposition et appropriation. Alterstice - Revue international de la recherche interculturelle, 8(1). Repéré à https://www.journal.psy.ulaval.ca/ojs/index.php/ARIRI/article/view/ orrissette_Alterstice $8 \% 281 \% 29$

MORRISSETTE, J., DIÉDHIOU, B. et CHARARA, Y. (2014). Un portrait de la recherche sur l'intégration socioprofessionnelle des enseignants formés à l'étranger [Rapport de recherche déposé au Centre d'intervention pédagogique en contexte de diversité de la Commission scolaire Marguerite-Bourgeois]. Montréal, Québec: Centre d'intervention pédagogique en contexte de diversité, Commission scolaire Marguerite-Bourgeoys. Repéré à https://www.cipcd.ca/wp-content/ uploads/2014/10/Rapport-Morrissette-et-al-vF-20-oct-2014.pdf

MORRISSETTE, J., MOTTIER LOPEZ, L. et TESSARO, W. (2012). La production de savoirs négociés dans deux recherches collaboratives sur l'évaluation formative. Dans L. Mottier Lopez et G. Figari (dir.), Modélisations de l'évaluation en éducation. Questionnements épistémologiques (p. 27-43). Bruxelles, Belgique: De Boeck. Repéré à http://citeseerx.ist.psu.edu/viewdoc/ download?doi=10.1.1.1019.8623\&rep=rep1\&type=pdf

MUJAWAMARIYA, D. (2008). Les difficultés d'insertion professionnelle des enseignants de sciences de race noire en milieu francophone ontarien: un pattern singulier? Dans L. Portelance, J. Mukamurera, S. Martineau et C. Gervais (dir.), L'insertion dans le milieu scolaire. Une phase cruciale du développement professionnel de l'enseignant (p. 145-161). Québec, Québec: Presses de l'Université Laval.

MYLES, J., CHENG, L. et WANG, H. (2006). Teaching in elementary school: Perceptions of foreign-trained teacher candidates on their teaching practicum. Teaching and Teacher Education, 22(2), 233-245. 
NIYUBAHWE, A., MUKAMURERA, J. et JUTRAS, F. (2013). Professional integration of immigrant teachers in the school system: A literature review. McGill Journal of Education, 48(2), 279-296.

PEELER, E. et JANE, B. (2005). Mentoring: Immigrant teachers bridging professional practices. Teaching Education, 16(4), 325-336.

PHILLION, J. (2003). Obstacles to accessing the teaching profession for immigrant women. Multicultural Education, 1(11), 41-45.

REMENNICK, L. (2002). Survival of the fittest: Russian immigrant teachers speak out about their professional adjustment in Israel. International Migration, 40(1), 99-121.

ROSS, F. (2001). Helping immigrants become teachers. Educational Leadership, 28(8), 68-71. Repéré à http://www.ascd.org/publications/educational-leadership/may01/ vol58/num08/Helping-Immigrants-Become-Teachers.aspx

SCHMIDT, C. (2010). Systemic discrimination as a barrier for immigrant teachers. Diaspora, Indigenous, and Minority Education, 4(4), 235-252.

WALSH, S. et BRIGHAM, S. (2008). Internationally educated teachers and teacher education programs in Canada: Current practices. Repéré à http://community. smu.ca/atlantic/documents/WP18--WalshandBrigham2.pdf

ZIETSMA, D. (2010). Immigrants exerçant des professions réglementées (n. 75-001-X). Ottawa, Ontario: Statistique Canada.

ZOLESIO, E. (2013). La socialisation chirurgicale, un apprentissage "par claques». Revue française de pédagogie, 184(3), 95-104. 